\title{
Design of a Passive Gait-based Ankle-foot Exoskeleton with Self-adaptive Capability
}

\author{
Xiangyang Wang ${ }^{1}$, Sheng Guo ${ }^{2 *}$, Bojian $\mathrm{Qu}^{3}$, Majun Song ${ }^{1}$ and Haibo Qu ${ }^{2}$
}

\begin{abstract}
Propulsion during push-off is the key to realizing human locomotion. Humans have evolved a way of walking with high energy utilization, but it can be further improved. Drawing inspiration from the muscle-tendon unit, a passive spring-actuated ankle-foot exoskeleton is designed to assist with human walking and to lengthen walking duration by mechanically enhancing walking efficiency. Detection of the gait events is realized using a smart clutch, which is designed to detect the contact states between the shoe sole and the ground, and automatically switch its working state. The engagement of a suspended spring behind the human calf muscles is hence controlled and is in synchrony with gait. The device is completely passive and contains no external power source. Energy is stored and returned passively using the clutch. In our walking trials, the soleus electromyography activity is reduced by as much as $72.2 \%$ when the proposed ankle-foot exoskeleton is worn on the human body. The influence of the exoskeleton on walking habits is also studied. The results show the potential use of the exoskeleton in humans' daily life.
\end{abstract}

Keywords: Ankle-foot exoskeleton, Energy cost, Self-adaptiveness, Human augmentation

\section{Introduction}

Legs are important for locomotive ability, and enable us to move and travel in our daily life. Enduring walking and lower energy costs can significantly expand the range of human activities. In some rough terrains, walking is the only option because wheeled vehicles are unable to transport people. Thus, improved walking economy is beneficial to everyone. Although the walking behavior of humans is well-tuned under natural selection [1], there is still room for improvement, especially when exoskeleton devices are developed and enter into service.

Since half of the required mechanical power output is generated by the ankle during push-off [2], the ankle-foot exoskeleton has been extensively studied over the past two decades. Many powered and unpowered devices have been proposed to assist in the push-off process, reducing the energy cost to the human body [3]. Although

\footnotetext{
*Correspondence: shguo@bjtu.edu.cn

2 Key Laboratory of Vehicle Advanced Manufacturing, Measuring and Control Technology, Ministry of Education, Beijing Jiaotong University, Beijing 100044, China

Full list of author information is available at the end of the article
}

ankle-foot orthoses (AFOs) are also capable of providing assistance during walking, they are often designed for people suffering from a leg pathology to increase their ambulatory ability, such as the MIT AFO [4].

Unlike AFOs, the ankle-foot exoskeleton (AFE) is a device that augments the performance of ankle movements by an able-bodied wearer [5]. In general, such devices can be divided into two categories according to the actuation mode: active ankle-foot exoskeleton (AAFE) and passive ankle-foot exoskeleton (PAFE).

AAFEs employ an external power source to provide biologically equivalent levels of joint power, and reduce the essential amount of energy required from human muscles [6]. Some clever mechanisms are designed so that the driving force of the motor can be converted into an assistive torque about the ankle joint. For example, Mooney et al. designed an autonomous exoskeleton that adopts a triangular structure formed by struts. Driven by a winch actuator, the device converts the pulling force into a torque about the ankle joint [7, 8]. Liu et al. [9] proposed a lightweight exoskeleton with an elastic spring that can continuously store the energy injected by a lightweight motor, and release it quickly to provide 
high-powered assistance. To achieve linear motion, Meijneke et al. [10] presented a series elastic actuator (SEA) consisting of an electric motor and a ball-screw gear to control the distance between two rigid endpoints on the shank and foot, respectively. Compared with electric motors, lightweight and the structural similarity with a muscle-tendon unit, greatly allow for the extensive application of pneumatic muscles in the design of the AAFE, such as the exoskeletons developed for the elderly by Galle [11, 12], and Ferris [13-16]. Although the mechanical structures of the devices mentioned above are sufficiently light, the energy cost of the combined mass of actuators and power sources is more than four times that of the same mass attached to the waist [17]. Therefore, the strategy of placing the actuators on the waist or back has been employed to achieve better walking economy. One example is the AAFE designed by Jackson et al. [18, 19], which is powered by a flexible Bowden cable and a motor. Based on this device, Zhang $[20,21]$ proposed a control method to realize the optimization of assistance during walking.

Although AAFEs can provide as much energy as required, there are still some disadvantages. One major concern is that the force profiles and onset timing are gait-based. Therefore, accurate detection of the gait stages is critical. Electronic sensors must be used to detect all kinds of gait events. In addition, the exoskeleton must be sufficiently compliant to adapt to the full ankle-joint range of motion without impeding normal human movement during the swing phase. The inability to dorsiflex freely could impose a significant metabolic penalty [22]. The distal mass is another problem. Circuit-based sensor-control systems and power sources can result in additional system mass and lead to reduced wearing comfort. Shock while walking is also a side factor that may damage the circuit. Because of the above reasons, purely mechanical exoskeletons (PAFEs) have been developed as an alternative.

Totally different from the powered exoskeleton, PAFEs have recently been studied by some scholars. Previous work revealed the advantages of such devices, e.g., these require no power source and have a circuit-based control system. Moreover, since all the functions are realized mechanically, these devices are highly reliable and can provide continuous assistance. Elastic elements (e.g., springs and elastic cables) are usually employed to store energy from the human body, and then release this energy to make users move more quickly. As a result, walking efficiency is noticeably increased [23]. Farris et al. [24] designed an exoskeleton with a suspended spring parallel to the calf muscles. However, owing to the lack of gait identification, the resting length of the spring must be adjusted for each participant.
To identify the gait period without using electronic sensors, smart clutches [1,22, 25-27] have been designed to mechanically control the engagement of the elastic components and provide assistance at the proper time. The clutch ensures that assistance is always provided at the right time without impeding the free movement of the ankle joint during the swing phase. Collins et al. [1, 22] designed a clutch based on the ratchet-pawl mechanism so that the spring linkage can be controlled by setting the timing of pawl latch and release. However, this device lacks adaptability as it needs to be customized for individuals. In another research by his team, an electro-adhesive clutch $[25,26]$ was presented to achieve the same purpose, which was actually quasi-passive. Recently, Yandell et al. [27] proposed a low-profile PAFE with a clutch that fits under clothes. However, the clutch may fail to be clutched if the stiffness of the spring is large enough. To solve these problems, a novel two-input clutch was presented in our previous work [28] with springs triggering the clutched state.

However, our previous design has a weakness. A timedelay problem occurs during state-switching due to the deformation of the trigger spring. The clutch only works normally when the users walk at a low speed. The problem tends to become more obvious as walking speed increases. In this paper, we perform an iteration based on our previous design. Walking trials are carried out based on the prototype to evaluate and verify the assistive performance of the newly proposed ankle-foot exoskeleton. The new clutch uses rigid instead of soft triggers to achieve faster state-switching. Compared with the existing clutch structure, the proposed clutch is suitable for almost all users and is reliable in maintaining a clutched state even though a large tensile force is generated in the rope attached to the clutch.

An energy conversion model is also developed to theoretically validate the feasibility of metabolic cost reduction when the PAFEs are used. This part of the work has not been verified by mathematics in the past. Although many walking trials in previous research have shown that human metabolic cost is reduced by wearing a PAFE, a theoretical model must be established and validated before the design process.

\section{Biomechanics and Energetics}

\subsection{Biomechanics during Human Walking}

A complete gait cycle can be divided into four gait stages according to the contact states between the shoe sole and the ground: the heel strike (HS), flat foot (FF), push-off $(\mathrm{PO})$, and swing. For the ankle joint, it generates positive or negative mechanical work during different gait periods (e.g., negative work during FF, and positive work during PO [28]). 
Both positive and negative work impose an energy cost on the human body [29]. Positive work is usually performed to assist the trunk as it vaults over the stance limb [30], whereas negative work is done with mechanical energy (ME) converted into other forms of energy. The reason for the negative part can be the energy stored in the tendons or dissipated owing to the damped motion of fat, viscera, and muscle [31]. Hence, only a part of the energy can be recycled during normal walking, with most of the energy wasted. Consequently, humans need to consume their biomass energy continuously to maintain their walking speed and compensate for the energy loss in the body due to dissipation.

Since energy stored in an elastic component can be returned almost without any loss, using a spring to reduce the dissipated part of the energy consumed by human tissues may be a good way to reduce human energy expenditure.

\subsection{Energy Conversion Model}

To demonstrate the feasibility of energy cost reduction using elastic components, a mathematic model is developed to compare the cases of normal walking and assisted walking with flexible components spanning the ankle joint. As shown in Figure 1, the skeletons in cases (a) and (b) undergo the same walking process (i.e., the initial ME at the initial state, final ME at the end state; the joints' trajectories are the same in both cases). A spring parallel to the calf muscles is attached to the human body with its two ends anchored at the shank and the foot. Our model focuses on the ankle dorsi/plantar flexion muscle group. The energy consumed in other segments of the human body is assumed the same in both cases.

One of the legs is considered, where negative work is done as the ankle dorsiflexes from the HS to the end stance. Part of the initial ME, which has a total amount of $x$, is converted and goes to two places, i.e., converted into elastic potential energy in the series elastic muscletendon mechanics [32], or dissipated in biological tissue. Bioenergy is continuously consumed from human body and injected into the kinematic system (KS) so that the final ME will not decrease gradually with step-to-step transitions.

Plantar flexor muscles (i.e., soleus, gastrocnemius, tibialis anterior) are the main muscle group that contributes to walking. Assuming that the proportional relationship between stored and dissipated energy in the plantar flexor muscles is fixed, for every unit of negative work done by the ankle joint, the stored part is $p(0<p<1)$, and there is a $(1-p)$ unit of energy dissipation. In case (a), the ME

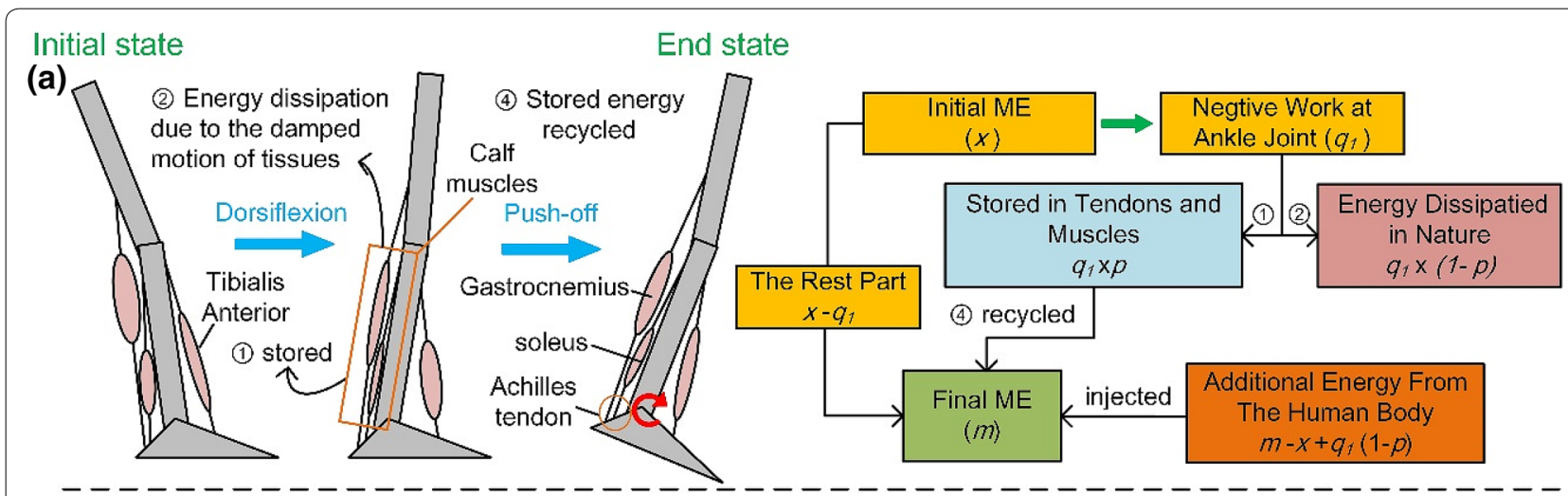

(b)

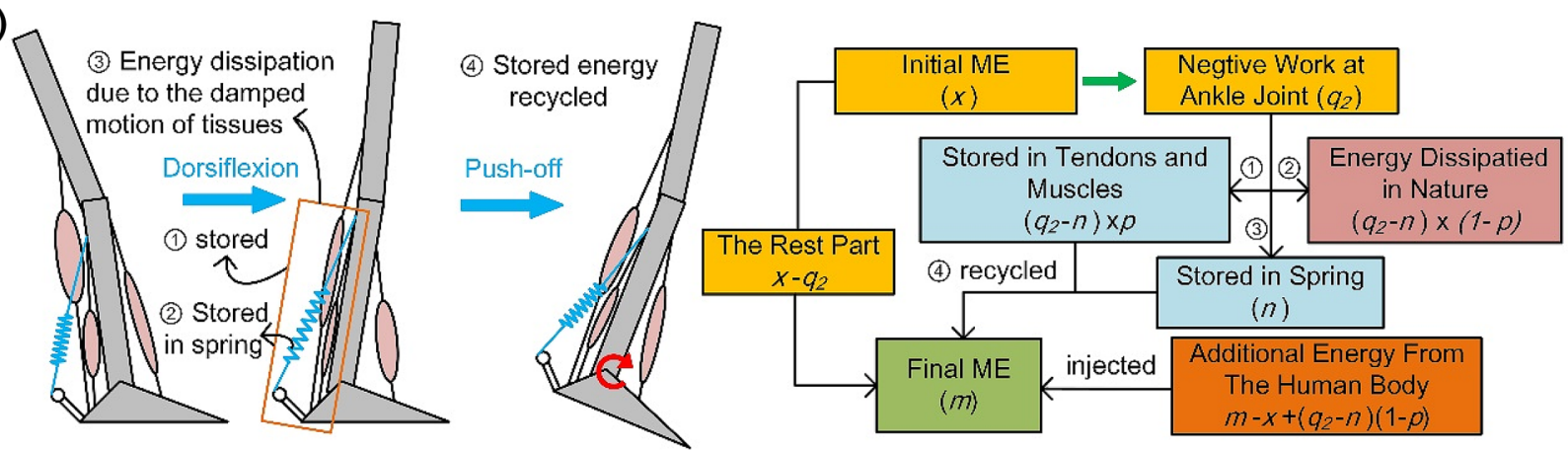

Figure 1 Energy conversion model: a Walking without the elastic component. b Walking with the elastic component, (both cases experience the same walking process from $\mathrm{HS}$ to $\mathrm{PO}$, where the initial ME and final ME in both cases are the same) 
of a total amount of $q_{1}$ is converted as the ankle outputs negative work, with $x-q_{1}$ left in the KS. The amount of energy stored in the muscle-tendon is $q_{1} \times p$, while the rest $q_{1} \times(1-p)$ dissipates simultaneously. During the $\mathrm{PO}$, the stored energy is returned. Additional energy in the amount of $m-x+q_{1}(1-p)$ must be injected into the KS from the human body, so that the desired movement can be achieved.

For case (b), in addition to the energy stored in the muscle-tendon unit, the spring is also stretched with quantities of ME converted into strain energy. The stiffer the spring, the more ME is converted. The total amount of $\left(q_{2}-n\right) \times p+n$ is thus recycled during PO. It can be derived that the total amount of $m-x+\left(q_{2}-n\right)(1-p)$ must be additionally provided by the human body. Compared with case (a), case (b) would save more energy when the following condition is satisfied:

$$
q_{2}-q_{1}<n
$$

Since part of the ME that should have been stored in the muscle-tendon unit is stored in the spring instead, as shown in the blue block in both cases in Figure 1, this implies

$$
q_{1} \times p>\left(q_{2}-n\right) \times p,
$$

Eq. (1) is thus satisfied.

Compared with normal walking, walking with elastic elements can save the human energy cost of $\left(q_{1}-q_{2}+n\right)(1-p)$. The amount relies on the proportional factor $p$ and the spring stiffness. Usually, it is impossible to alter the factor $p$ since it is a physiological property of individuals. However, we can increase the proportion of energy stored in the spring, i.e., the factor $n$, so that the metabolic cost to the human body can be reduced.

It's worth noting that $n$ must be less than $x$. In general, the ME conversion process during dorsiflexion will slow the pendular motion of the human body. A spring that is too stiff will impede the normal walking process and result in reduced wearing comfort.

\section{Design Overview}

\subsection{Goals}

The main purpose of this research is to design a PAFE with a smart clutch based on our previous work [28]. The device is intended to address the problem of time-delay, and perform the function of automatic state-switching based on mechanical identification of the current gait stages. The engagement of the elastic spring relies on the clutch state, and controls the energy storage and release process without impeding the free ankle motion during the swing phase. The device aims to reduce the metabolic expenditure of human body without any power sources, motors, or electronic sensors.

\subsection{General Overview}

Figure 2 presents an overview structure of the PAFE with the newly designed clutch. It consists of four parts: the clever clutch, an extension spring, a shank brace, and a shoe. The clutch is fixed at the outer side of the shoe sole and mounted on a $10 \mathrm{~mm}$ thick $\mathrm{Al}$-alloy plate. The $\mathrm{Al}$ alloy plate is bolted with a shoe sole, of which half the heel is cut off from the backside for the Al-alloy plate so that wearers can keep their foot flat when standing normally.

One end of the wire rope is attached to a pulley in the clutch, whereas the other end is routed to the back of the human foot via three U-groove bearings and connected to the lower end of the extension spring. The shank brace comprises a plastic shell and strap. The inside of the plastic shell is filled with sponges to improve wearing comfort. The strap is made of nylon to prevent deformation when force is applied. The proposed PAFE has no hingelike artificial joint, and nonsagittal plane motion can be achieved without restraints.

The upper end of the extension spring is attached to an anchoring point at the backside of the shank brace. The spring is supposed to be engaged at the beginning of the HS when the clutch is clutched and stretched to its maximum length at the terminal stance with a large restoring force. During the PO, the spring releases the stored energy and provides assistive ankle moment, propelling the human body to move forward, thus reducing muscle force. During the swing phase, the extension spring is supposed to be disengaged when the clutch is switched to an unclutched state, which ensures the free movement of the ankle joint.

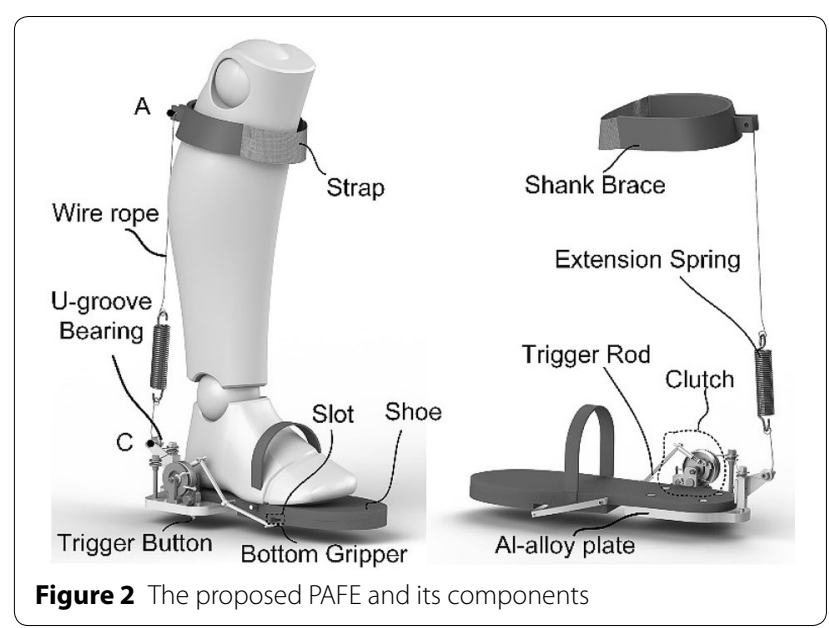




\subsection{Clutch Design}

Considering that the foot is "rolling" from the rear foot to the forefoot (see Figure 3), we propose a double-trigger method to distinguish corresponding gait stages. A trigger rod and trigger button are separately distributed at the foresole and rearsole. They can be moved under the ground reaction force (GRF), pushing other components in the clutch to achieve mechanical control of the clutch states.

The clutch is the core component of the exoskeleton. The proposed clutch has a small size and consists of many tiny components. Figure 4a shows an enlarged view of the internal structure of the clutch. Shaft seat (a) and (b) are mounted on the plate. The pulley and connecting rod (b) are concentric with the shaft (a) passing through their center holes. The lever near the connecting rod (b) is threaded onto the lever holder that is bolted to the shaft seat (a). The return spring (c) is used to help the lever revert to its original position when the human foot is off the ground. At the original position, there is no contact between the rubber block and pulley. The connecting rod (b) and lever are connected by the connecting rod (a). A four-bar mechanism is thus formed.

When the foresole touches the ground, the forced motion of the trigger rod can be transmitted by a transmission mechanism and the four-bar mechanism, resulting in contact between the flange of the pulley and the rubber block attached to the lever. The transmission mechanism consists of an upper rod and a lower rod used for length adjustment so that the GRF can be transmitted into the clutch as the bottom gripper is compressed into the U-shape slot at the foresole (see Figure $4 \mathrm{~b}$ ). Wearing comfort is thus improved.

Similarly, the trigger button pushes the pin (a) to move upward when the rearfoot touches the ground. This leads to the clockwise motion of the lever about the lever holder and results in contact between the rubber block and pulley. The return spring (a) is placed outside the trigger button with its two ends keeping contact with the shaft shoulder of the trigger button and the inner shoulder of the bottom block,

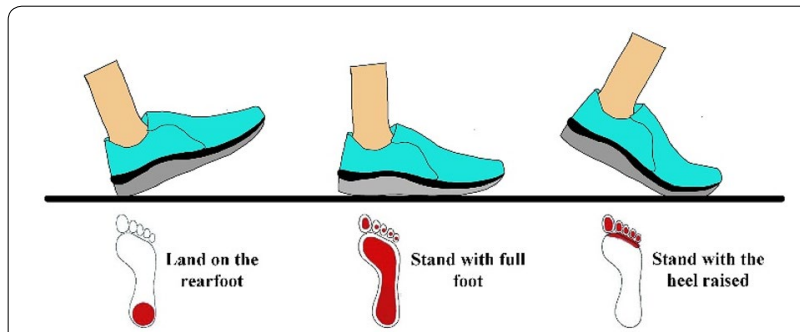

Figure 3 Schematics of contact between the ground and different areas of the human foot at different walking periods respectively, so that the trigger button can be moved to its original position when no GRF is applied.

Friction is generated between the rubber block and pulley flange as contact occurs, resisting the clockwise rotation of the pulley. Deformation of the rubber block occurs under the action of friction and results in a much larger friction force due to the increased normal stress perpendicular to the contact surface. The clutch is therefore clutched with the clockwise rotation limited. The two inputs, represented as red arrows in Figure 5 , are independent in triggering the clutched state of the clutch.

As the clutch is clutched, the wire rope cannot be dragged out from the pulley. Consequently, the lower end of the extension spring is fixed, and the spring can be stretched as the foot dorsiflexes during the FF with energy stored. Until the foot is off the ground, the clutch is unclutched, and the pulley is allowed to rotate in both directions. The rope can be freely dragged in and out of the pulley without impeding the free rotation of the biological ankle joint.

To keep the cord tensioned throughout the gait cycle, a constant force spring is placed on the spring holder. It is attached to the edge of the pulley and always generates a constant counterclockwise torque, which can apply a constant tensile force on the rope. When the rope is slack, or the operating length (distance between the points $\mathrm{A}$ and $\mathrm{C}$ in Figure 2) decreases, the excess rope will be dragged into the pulley immediately.

\subsection{Prototype Specification}

A prototype has been manufactured and assembled. The thickness of the pulley flange is $1 \mathrm{~mm}$, and the constant spring can provide a pulling force of $8 \mathrm{~N}$. The metal plate and linkage mechanisms are made of $6061 \mathrm{Al}$-alloy, while all the shafts are made of stainless steel. The material of the shoe is rubber. The shank brace was made from a leg guard often used by football players as it is lightweight, and its curved surface fits the users' leg size. The total weight of our prototype is $765.5 \mathrm{~g}$ (shoe included), of which $349 \mathrm{~g}$ is due to the Al-alloy plate, $42 \mathrm{~g}$ is due to the shank brace, and $46.2 \mathrm{~g}$ is due to the extension spring. A comparison was made between our PAFE and the previous ones listed in Table 1. It shows that AAFE is generally heavier than those without power sources. Among the passive devices, our prototype seems slightly heavier than those developed by Collins and Yandell. However, the weight of our prototype can be further reduced when lightweight materials are used. The Al-alloy plate is 10 $\mathrm{mm}$ thick, and occupies a large proportion (45.6\%) of the overall mechanical device. 


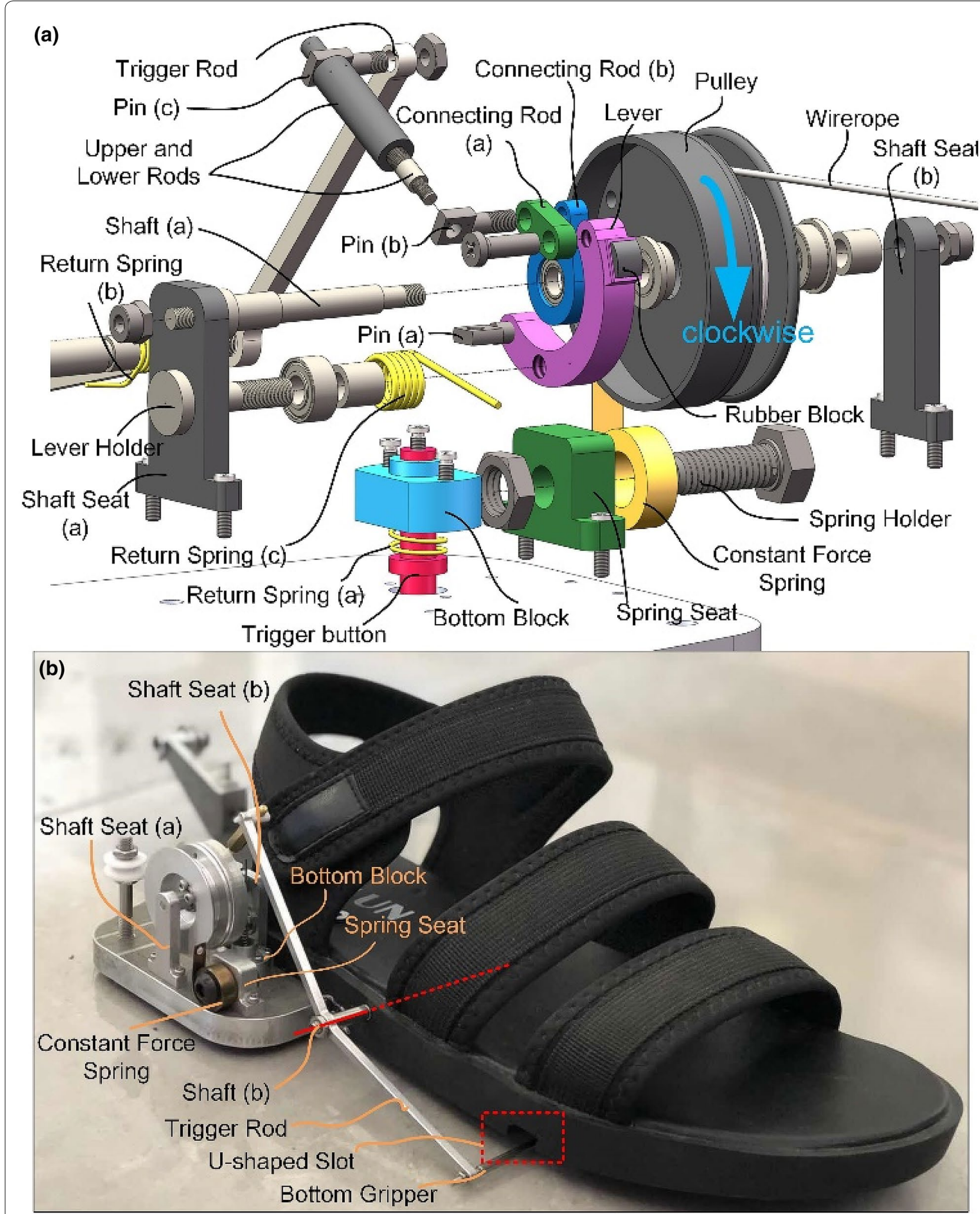

Figure 4 a Schematics showing the assembly structure of the clever clutch. b Prototype of the proposed PAFE 


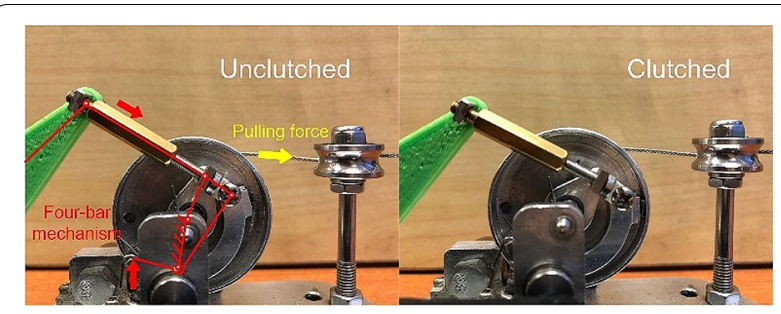

Figure 5 Clutched and unclutched states of the proposed clutch (it relies on the contact states between the rubber block and the flange of the pulley)

\section{Walking Trials}

\subsection{Performance Evaluation}

To evaluate the assistance provided by our PAFE to the plantarflexion muscles, five volunteers participated in walking trials (all males aged 22-46 years old). All procedures in this study were carried out in accordance with the protocol approved by the Institutional Review Board of Beijing Jiaotong University. All the human subjects gave their written informed consent prior to participation.

First, subjects walked on a treadmill in casual shoes at $4 \mathrm{~km} / \mathrm{h}$ for $1 \mathrm{~min}$ without a break. Then, the speed was increased to $5 \mathrm{~km} / \mathrm{h}$, and the walking test was repeated. The subjects were required to rest for $10 \mathrm{~min}$ between consecutive sets of gait tests. Both tests served as control. Then, they walked while wearing the PAFE on their right leg and repeated the tests at the same speeds. Since walking speed relies on the stride length and gait frequency, the subjects were required to keep their walking pace constant (i.e., frequency is almost fixed) during the test, and their stride length consequently varied. Two sets of spring stiffness were used in the assistive walking to verify the performance (low stiffness: $1.58 \mathrm{~N} / \mathrm{mm}$ and high stiffness: $9.05 \mathrm{~N} / \mathrm{mm}$ ). The electromyography (EMG) of the soleus of each subject was recorded during $1 \mathrm{~min}$ of walking. Two electrodes were attached to the soleus (see Figure 6), whereas the reference electrode was attached to the patella, where the muscle activity is extremely weak so that the calibration process is easier. After lowpass filtering, rectification, and amplification, the EMG signal could be read directly.

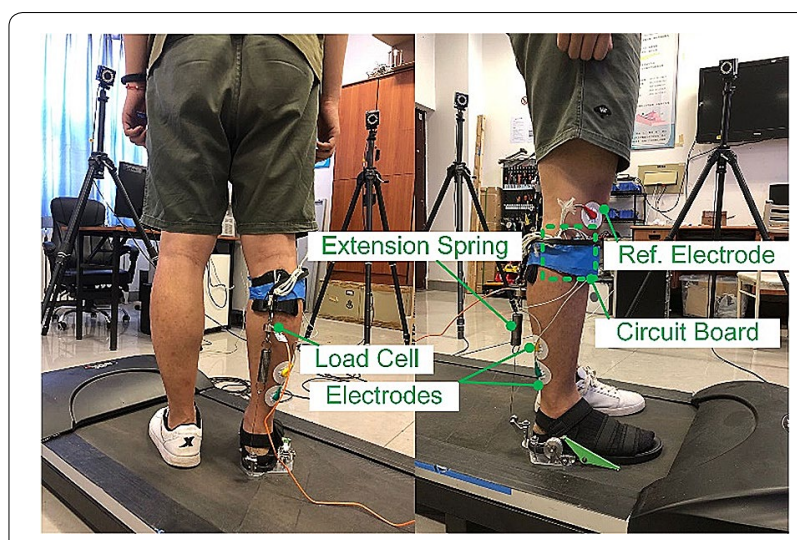

Figure 6 Walking trials and the sensors used to record biological and mechanical signals

Figure 7 shows the soleus activity of an arbitrary three strides of one subject in the walking test. Compared with normal walking (without assistance), the maximum instantaneous muscle activity during the PO decreased when the PAFE was worn. At $4 \mathrm{~km} / \mathrm{h}$, however, the soleus was more active during the FF compared with normal walking when the low-stiffness spring was employed. This could be attributed to the additional mass attached to the human body and very limited assistance provided by the exoskeleton. In contrast, the spring with high stiffness provided relatively larger assistance. Consequently, muscle activity remained low throughout the gait cycle. In the swing phase, there was little difference in activity among these cases, since the spring was disengaged and had almost no influence on muscle activity.

As the walking speed increased, muscle activity increased accordingly as more energy is required from the human body to maintain a faster walking speed. Table 2 shows the comparison of the peak value of the muscle activity at different walking speeds. The magnitudes of the activities were averaged across three continuous strides. Comparatively speaking, the exoskeleton with high stiffness provides more assistance and results in a remarkable reduction in soleus activity. When a lowstiffness spring was used, less assistance was provided. The results also show increased percentage reduction of muscle activity under the same spring stiffness condition

Table 1 Mass comparison on recent ankle-foot exoskeletons (some data are collected by Yandell et al. [27])

\begin{tabular}{|c|c|c|c|c|c|c|c|c|}
\hline \multirow[b]{2}{*}{ Mass per leg (g) } & \multirow{2}{*}{$\begin{array}{l}\text { Mooney et al. [7] } \\
2300\end{array}$} & \multicolumn{2}{|c|}{$\begin{array}{l}\text { Witte et al. } \\
\text { [19] }\end{array}$} & \multirow{2}{*}{$\begin{array}{l}\text { Collins et al. [1] } \\
408-503\end{array}$} & \multirow{2}{*}{$\begin{array}{l}\text { Ferris et al. [14] } \\
1700\end{array}$} & \multirow{2}{*}{$\begin{array}{l}\text { Meijneke } \\
\text { et al. [10] }\end{array}$} & \multirow{2}{*}{$\begin{array}{l}\begin{array}{l}\text { Yandell } \\
\text { et al. [27] }\end{array} \\
598\end{array}$} & \multirow{2}{*}{$\begin{array}{l}\text { Wang et al. } \\
765.5\end{array}$} \\
\hline & & 835 & 875 & & & & & \\
\hline Mass at waist/back (g) & 1700 & 0 & 0 & 0 & 0 & 5200 & 0 & 0 \\
\hline Powered & Yes & No & No & No & Yes & Yes & No & No \\
\hline
\end{tabular}



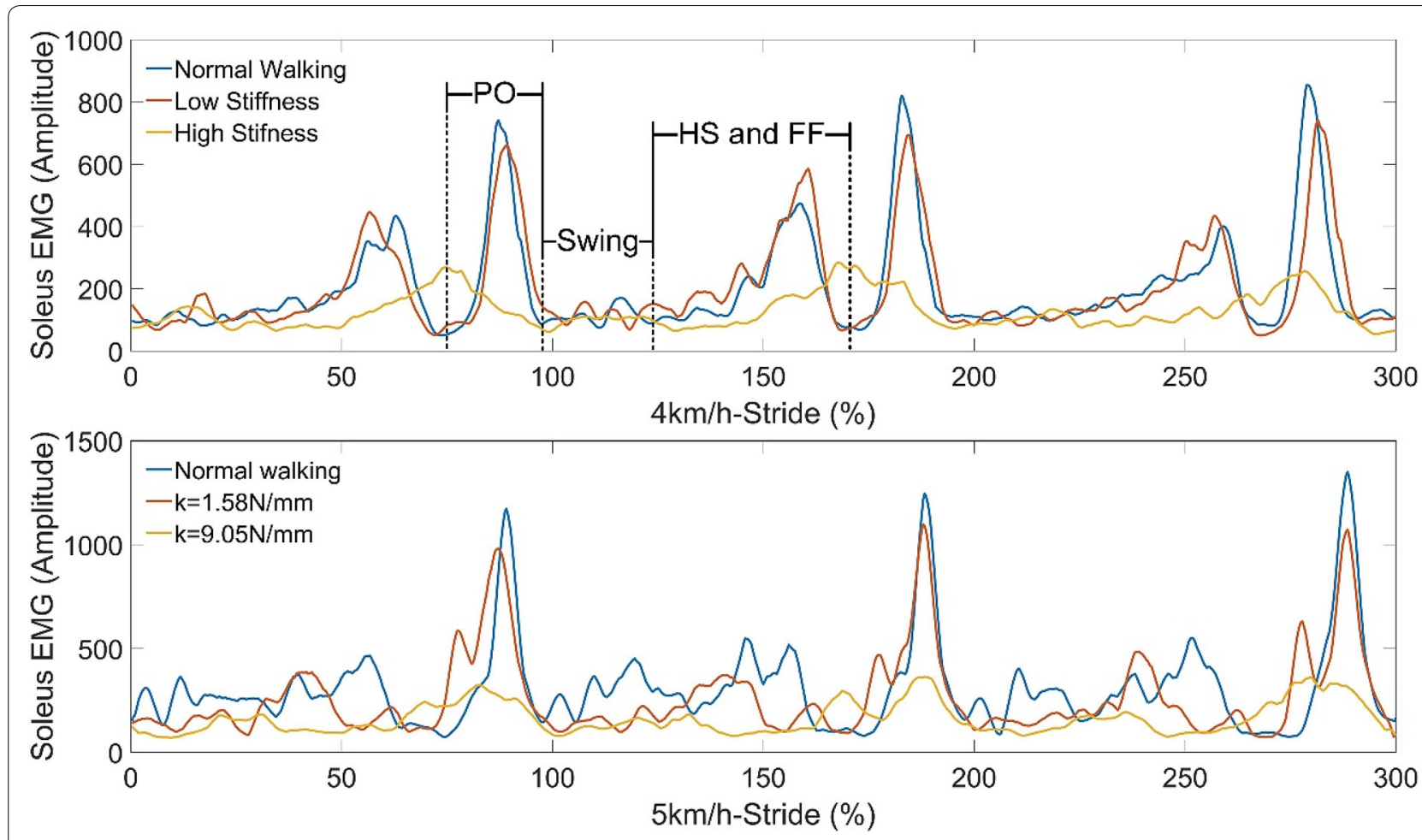

Figure 7 EMG signals of the soleus at different walking speeds and spring stiffness conditions

Table 2 Data comparison on EMG signals and spring forces

\begin{tabular}{llllll}
\hline & Normal walking & Low stiffness & Reduction & High stiffness & Reduction \\
\hline $4 \mathrm{~km} / \mathrm{h}$ & E: 804.8 & E: 699.0 & E: $13 \%$ & E: 269.6 & E: $66.5 \%$ \\
& T: $29.7 \mathrm{~N}$ & & T: $101 \mathrm{~N}$ & \\
$5 \mathrm{~km} / \mathrm{h}$ & E: 1256.7 & E: 1050.6 & E: $16.4 \%$ & E: 349.4 & \\
& & T: $30.6 \mathrm{~N}$ & & T: $107.3 \mathrm{~N}$ & E: $72.2 \%$ \\
\hline
\end{tabular}

Note: E: EMG signal; T: tensile force

as the walking speed increased, because a longer step length results in larger spring stretching and restoring force.

In addition to the EMG signal, the assistive tensile force was also measured by a load cell serially connected with the spring. Figure $8 \mathrm{a}$ shows the measured tensile forces averaged across three gait cycles. The tensile force during swing is due to the pulling force (approximately $8 \mathrm{~N}$ ) of the constant force spring within the clutch. From the HS, the spring is continuously stretched, with energy slowly stored in the spring, and then released stably and quickly during the PO. The maximum spring forces measured (also known as the tensile force) are also listed in Table 2.

However, according to our calculations based on the data from the motion capture system (Cortex, Motion Analysis Co., USA), the operating length increased by 25 $\mathrm{mm}$ (at $5 \mathrm{~km} / \mathrm{h}$ ) and $24.3 \mathrm{~mm}$ (at $4 \mathrm{~km} / \mathrm{h}$ ) from its initial length at the HS to its maximum value as the ankle dorsiflexed. The increment is equal to the stretched length of the spring, of which the maximum restoring force can be calculated based on Hooke's law:

$$
F_{\text {max }}=k \cdot \Delta l \text {, }
$$

where $k$ denotes the stiffness of the spring.

The measured spring force differs from the calculated spring force $F_{\max }$ (defined as the reference spring force) under all stiffness conditions, as shown in Figure 8a. This can be attributed to the reduced operating length resulting from the deformation of biological tissues at the interface between the shank and brace. Since the spring force is held by the shank brace, slippage from its original position occurs. The actual stretched distance is smaller than the expected value. The stiffer the spring, the larger 


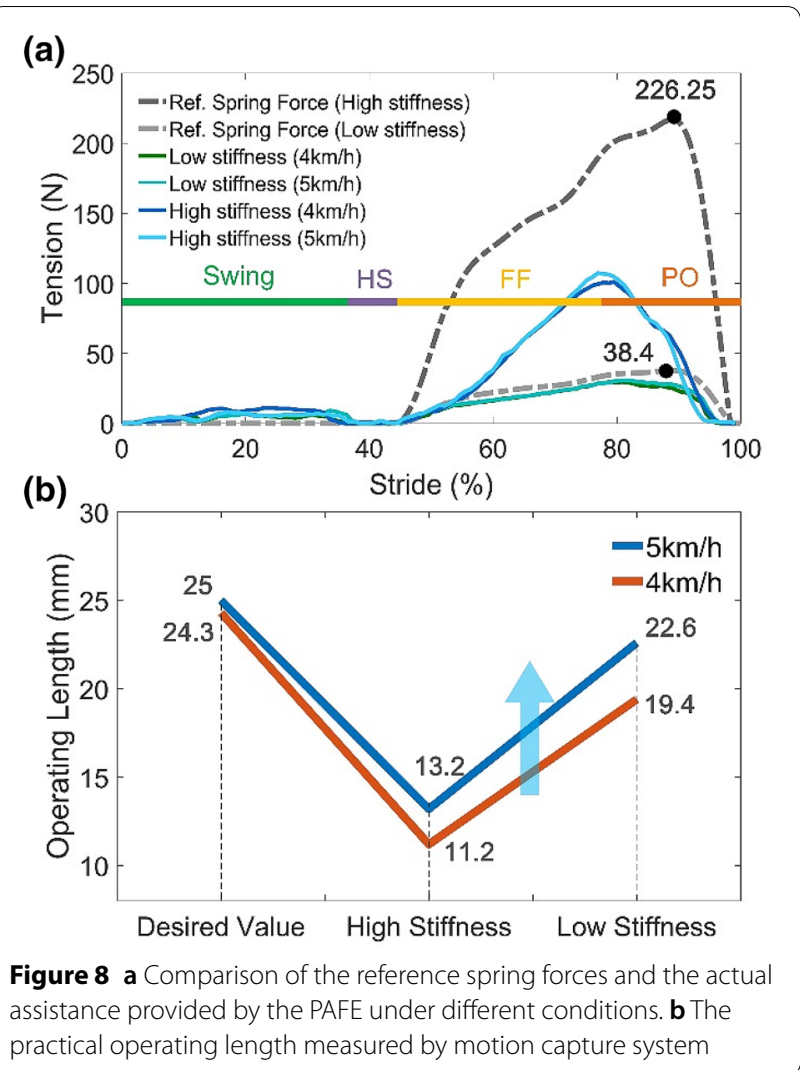

the generated deformation. Walking speed may also affect the slipping distance.

To verify this, two markers were attached to points A and $C$, respectively. The practical operating length can be measured with the motion capture system during the walking trials at different walking speeds. As shown in Figure $8 \mathrm{~b}$, the operating length decreased by approximately $50 \%$ when the high-stiffness spring was used, and by $7.0 \%-20.2 \%$ in the low-stiffness case as the walking speed gradually increased from $4 \mathrm{~km} / \mathrm{h}$ to $5 \mathrm{~km} / \mathrm{h}$. This is because a faster walking speed leads to a larger step length and larger ankle angle variation.

Slipping is a critical problem that leads to less assistance and benefits from the PAFE. We attempted to solve this problem by using more straps to affix the plastic shell of the shank brace. The slipping was reduced, and the generated spring force was comparatively larger. However, this caused lower wearing comfort. This problem can be further addressed by designing an improved human-machine interface; for example, a shank frame.

\subsection{Effects on Normal Gait}

It has been shown that the proposed PAFE can reduce the soleus force to a certain extent, but its influence on the walking habit is another concern.

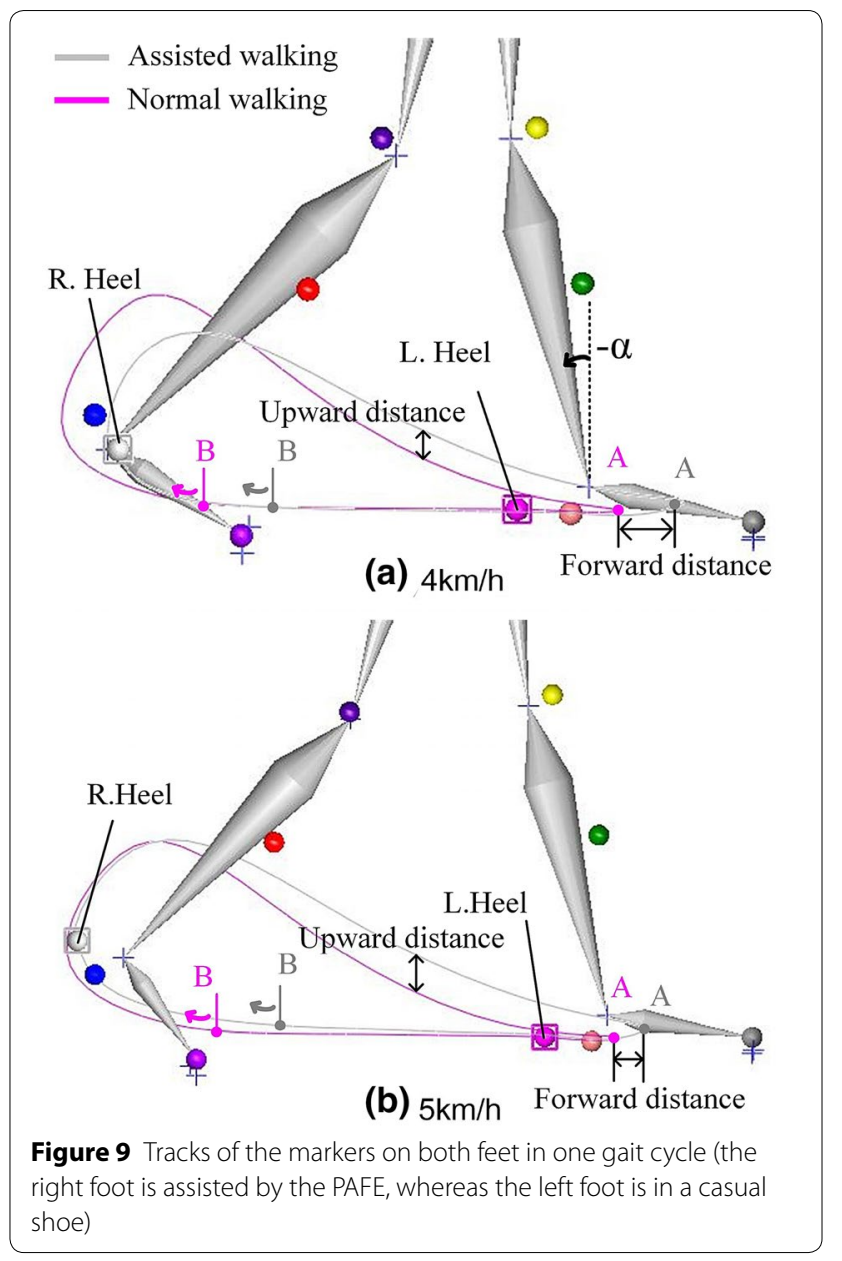

With the help of the motion capture system, we also examined the potential influence of the PAFE on human walking habits. Kinematic information was collected when the PAFE with high-stiffness spring was worn on the right foot. The left foot was in a casual shoe and regarded as a control group. A comparison can be made between the walking behavior of the two legs.

Figure 9 shows the plotted trajectories of the markers at the heel of both legs. Owing to the additional mass of the exoskeleton, the subject tends to consciously raise his right foot higher than his left foot. Also, the landing point A of the right foot is always further forward than the left foot, which is due to the introduced inertia of the exoskeleton. During the PO, the heel of the right foot is raised earlier than the left foot. As a result, the point $\mathrm{B}$ of the right foot, where the heel begins to leave the ground, is always ahead of the one of the left foot. This is due to the generated assistive torque about the ankle joint.

The ankle angles of both legs are shown in Figure 10. At $4 \mathrm{~km} / \mathrm{h}$, the average variations of the dorsiflexion angle $\alpha$ (defined in Figure 9) during the FF are $24.9^{\circ}$ (for normal 

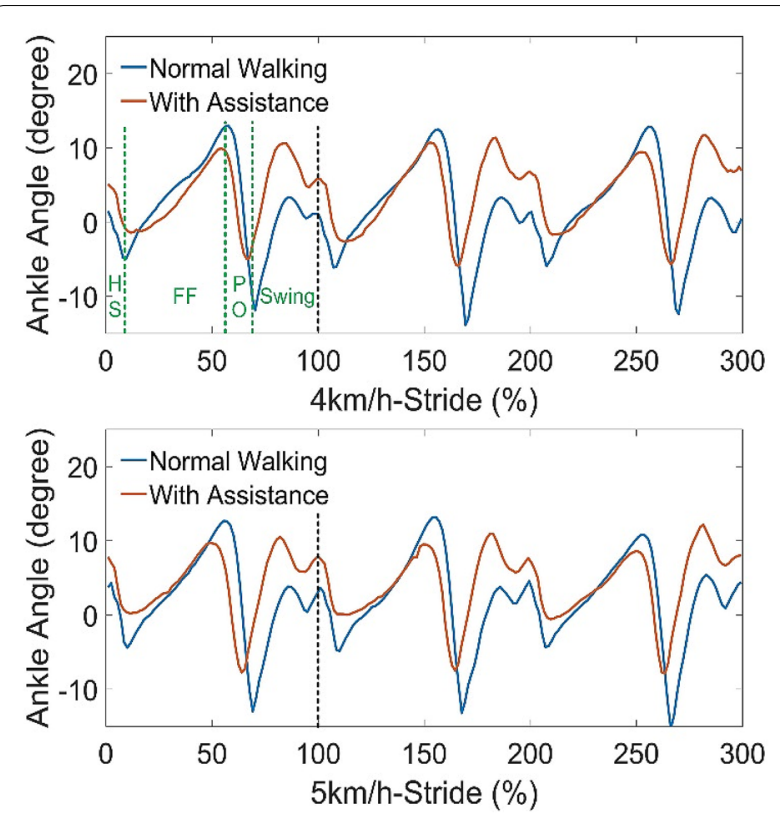

Figure 10 Comparison of the ankle angle between normal walking and assisted walking at different walking speeds

walking) and $14.9^{\circ}$ (for assisted walking). At $5 \mathrm{~km} / \mathrm{h}$, the angle variations are $25.6^{\circ}$ and $19.4^{\circ}$, respectively. With PAFE, the foot dorsiflexion was attenuated, which also led to a shorter stride length. During the swing phase, the ankle angle of normal walking was always less than that of assisted walking, with an average angle difference of $6.3^{\circ}$. An obvious "foot-drop" occurred when the PAFE was worn on the foot due to the additional gravitational torque generated by the device.

Subjectively speaking, the subject did not feel discomfort nor was he uncoordinated with the device. If a lighter material is used, then the influence on the normal gait can be further reduced.

\section{Limitations}

Although the proposed PAFE can provide favorable assistance during walking, the upper stiffness limit of the spring was not determined. The stiffness of the spring should be properly chosen so that the COM velocity of the human body will not drop to zero when it performs the pendular motion. A further experiment can be carried out to find a subjectively suitable stiffness range for users with different body weights and walking speeds. Another limitation is the slipping of the shank brace, which results in reduced tensile force. In our walking tests, the tensile force decreased by as much as $55.2 \%$ when the high-stiffness spring was used. The interface between the shank and shank brace must be redesigned to address this problem.

\section{Conclusions}

In this paper, we proposed a novel PAFE that is lightweight and can assist in the walking process by the mechanical identification of current gait stages, which is completely passive and suitable for nearly all users. Compared with our previous work, the newly designed clutch adopted rigid triggers so that the clutch state can be immediately changed as the GRF is transmitted into the clutch without any delay.

An energy conversion model was developed to validate the feasibility of our design in theory. Unlike previous research, a clever clutch was designed with two inputs that can identify the gait stages based on the contact status between the shoe sole and the ground. By introducing special mechanical constraints, the clutch can stay latched despite the application of an extremely large pulling force. The switching between the clutched and unclutched states is comparatively more reliable.

A prototype was produced based on the design. Walking trials were also carried out to evaluate the assistance provided by the PAFE under different speed and stiffness conditions. The muscle activity and spring forces were compared, which showed the benefit of the exoskeleton and its potential use in walking assistance. The influence of the PAFE on walking habits was also studied. With the assistance, the stride length was shortened along with possible foot-drop problems. However, these side effects can be addressed by practice walking and further reducing the device mass.

\section{Acknowledgements \\ All the authors would like to thank thank Yang Du for providing equipment support (Zebris FDM-T, Zebris Medical GmbH, Germany).}

\section{Authors' Contributions}

XW and SG were in charge of the whole trial and wrote the manuscript; BQ and MS were in charge of the prototype manufacturing and data analysis, $\mathrm{HQ}$ assisted with the use of software. All authors read and approved the final manuscript.

\section{Authors' Information \\ Xiangyang Wang, born in 1995, received his B.E. degree in Mechanical Engi- neering, in 2017, from Beijing Jiaotong University, China, where he is currently working toward the Ph.D. degree at Robotics Institute, School of Mechanical, Electronic and Control Engineering, Beijing Jiaotong University, China. His research interests include mechanical design and control of lower-limb exoskeletons.}

Sheng Guo, born in 1972, received his Ph.D. degree from Beijing Jiaotong University, China, in 2005. He was then a postdoctoral fellow at Cheng Kung University, Taiwan, China, in 2005-2006. He was a Visiting Scholar at the University of California, US, in 2010-2011. Currently, he is a Full Professor, Vice Director of Robotics Institute, and Vice Dean of the School of Mechanical, Electronic and Control Engineering, Beijing Jiaotong University. His research interests include robotics mechanism and mechatronics.

Bojian Qu, born in 1997, graduated from School of Mechanical and Electronic Control Engineering, Beijing Jiaotong University, with a bachelor's degree in mechanical engineering in 2019. He is currently working toward a master degree with his major research direction being automobile structural design and control at the KTH Royal Institute of Technology, Sweden. 
Majun Song, born in 1990, is currently a Ph.D. candidate at the Robotics Institute, School of Mechanical, Electronic and Control Engineering, Beijing Jiaotong University, China. He received his M.S. degree in Mechanical Engineering from Jiangxi University of Science and Technology, China in 2016, and the B.E. degree in Mechanical Engineering and Automation from Zhijiang College of Zhejiang University of Technology, Hangzhou, China, in 2013. His primary research interests focus on the design and control of prosthetic mechanisms.

Haibo Qu, born in 1984, received his Ph.D. degree from Beijing Jiaotong University, China, in 2013. Currently he is an Associate Professor at Robotics Institute, Beijing Jiaotong University. His research interests include parallel manipulator and mechanism theory.

\section{Funding}

Supported by Beijing Natural Science Foundation (Grant No. L172021), National Natural Science Foundation of China (Grant No. 51875033), and Fundamental Research Funds for the Central Universities (Grant No. 2019YJS164).

\section{Competing Interests}

The authors declare no competing financial interests.

\section{Author Details}

1 Robotics Institute, School of Mechanical, Electronic and Control Engineering, Beijing Jiaotong University, Beijing 100044, China. ${ }^{2}$ Key Laboratory of Vehicle Advanced Manufacturing, Measuring and Control Technology, Ministry of Education, Beijing Jiaotong University, Beijing 100044, China. ${ }^{3}$ School of Engineering Science, KTH Royal Institute of Technology, 10044 Stockholm, Sweden.

Received: 28 December 2019 Revised: 17 May 2020 Accepted: 2 June 2020

Published online: 17 June 2020

\section{References}

[1] S H Collins, M B Wiggin, G S Sawicki. Reducing the energy cost of human walking using an unpowered exoskeleton. Nature, 2015, 522(7555): 212.

[2] D J Farris, G S Sawicki. The mechanics and energetics of human walking and running: A joint level perspective. Journal of the Royal Society Interface, 2012, 9(66): 110-118.

[3] G S Sawicki, D P Ferris. Mechanics and Energetics of level walking with powered ankle exoskeletons. Journal of Experimental Biology, 2008, 211(9): 1402-1413.

[4] J A Blaya, H M Herr. Adaptive control of a variable-impedance ankle-foot orthosis to assist drop-foot gait. IEEE Transactions on Neural Systems and Rehabilitation Engineering, 2004, 12(1): 24-31.

[5] A M Dollar, H M Herr. Lower extremity exoskeletons and active orthoses: challenges and state-of-the-art. IEEE Transactions on Robotics, 2008, 24(1): 144-158.

[6] P Malcolm, W Derave, S Galle, et al. A simple exoskeleton that assists plantarflexion can reduce the metabolic cost of human walking. PLOS ONE, 2013, 8(2): e56137.

[7] L M Mooney, E J Rouse, H M Herr. Autonomous exoskeleton reduces metabolic cost of human walking during load carriage. Journal of NeuroEngineering and Rehabilitation, 2014, 11(1): 80.

[8] L M Mooney, H M Herr. Biomechanical walking mechanisms underlying the metabolic reduction caused by an autonomous exoskeleton. Journal of NeuroEngineering and Rehabilitation, 2016, 13(1): 4.

[9] J Liu, C Xiong, C Fu. An Ankle Exoskeleton using a lightweight motor to create high power assistance for push-off. Journal of Mechanisms and Robotics, 2019, 11(4): 041001.

[10] C Meijneke, WV Dijk, HV D Kooij. Achilles: an autonomous lightweight ankle exoskeleton to provide push-off power. Proc. 5th IEEE RAS/EMBS International Conference on Biomedical Robotics and Biomechatronics, Sao Paulo, Brazil, IEEE, 2014.

[11] S Galle, W Derave, F Bossuyt, et al. Exoskeleton plantarflexion assistance for elderly. Gait and Posture, 2017, 52: 183-188.
[12] S Galle, P Malcolm, W Derave, et al. Enhancing performance during inclined loaded walking with a powered ankle-foot exoskeleton. European Journal of Applied Physiology, 2014, 114(11): 2341-2351.

[13] D P Ferris, J M Czerniecki, B Hannaford. An ankle-foot orthosis powered by artificial pneumatic muscles. Journal of Applied Biomechanics, 2005, 21(2): 189-197.

[14] D P Ferris, K E Gordon, G S Sawicki, et al. An improved powered anklefoot orthosis using proportional myoelectric control. Gait and Posture, 2006, 23(4): 425-428.

[15] P Kao, C L Lewis, D P Ferris. Invariant ankle moment patterns when walking with and without a robotic ankle exoskeleton. Journal of Biomechanics, 2010, 43(2): 203-209.

[16] G S Sawicki, KE Gordon, D P Ferris. Powered lower limb orthoses: applications in motor adaptation and rehabilitation. Proc. International Conference on Rehabilitation Robotics, Chicago, IL, USA, 2005: 206-211.

[17] R C Browning, J R Modica, R Kram, et al. The effects of adding mass to the legs on the energetics and biomechanics of walking. Medicine and Science in Sports and Exercise, 2006, 39(3): 515-525.

[18] RW Jackson, S H Collins. An experimental comparison of the relative benefits of work and torque assistance in ankle exoskeletons. Journal of Applied Physiology, 2015, 119(5): 541-557.

[19] K A Witte, J Zhang, R W Jackson, et al. Design of two lightweight, highbandwidth torque-controlled ankle exoskeletons. Proc. International Conference on Robotics and Automation, Seattle, WA, USA, IEEE, 2015 1223-1228.

[20] J Zhang, P Fiers, K A Witte, et al. Human-in-the-loop optimization of exoskeleton assistance during walking. Science, 2017, 356(6344): 1280-1284.

[21] J Zhang, C Cheah, S H Collins. Experimental comparison of torque control methods on an ankle exoskeleton during human walking. Proc. International Conference on Robotics and Automation, Seattle, WA, USA, IEEE, 2015: 5584-5589.

[22] M B Wiggin, G S Sawicki, S H Collins. An exoskeleton using controlled energy storage and release to aid ankle propulsion. Proc. International Conference on Rehabilitation Robotics, Zurich, Switzerland, IEEE, 2011: 12178956.

[23] A M Grabowski, H M Herr. Leg exoskeleton reduces the metabolic cost of human hopping. Journal of Applied Physiology, 2009, 107(3): 670-678.

[24] D J Farris, G S Sawicki. Linking the mechanics and energetics of hopping with elastic ankle exoskeletons. Journal of Applied Physiology, 2012, 113(12): 1862-1872.

[25] S Diller, C Majidi, S H Collins. A lightweight, low-power electroadhesive clutch and spring for exoskeleton actuation. Proc. International Conference on Robotics and Automation, Stockholm, Sweden, IEEE, 2016: 682-689.

[26] S B Diller, S H Collins, C Majidi. The effects of electroadhesive clutch design parameters on performance characteristics. Journal of Intelligent Material Systems and Structures, 2018, 29(19): 3804-3828.

[27] M B Yandell, J R Tacca, K E Zelik. Design of a low profile, unpowered ankle exoskeleton that fits under clothes: overcoming practical barriers to widespread societal adoption. IEEE Transactions on Neural Systems \& Rehabilitation Engineering, 2019, 27(4): 712-723.

[28] X Wang, S Guo, B Q, et al. Design of a purely mechanical sensor-controller integrated system for walking assistance on an ankle-foot exoskeleton. Sensors, 2019, 19(14): 3196.

[29] P H Tzu-wei, A D Kuo. Mechanics and energetics of load carriage during human walking. Journal of Experimental Biology, 2014, 217(4): 605-613.

[30] G S Sawicki, C L Lewis, D P Ferris. It pays to have a spring in your step. Exercise and Sport Sciences Reviews, 2009, 37(3): 130-138.

[31] A D Kuo, J M Donelan, A Ruina. Energetic consequences of walking like an inverted pendulum: Step-to-step transitions. Exercise and Sport Sciences Reviews, 2005, 33(2): 88-97.

[32] G S Sawicki, N S Khan. A simple model to estimate plantarflexor muscletendon mechanics and energetics during walking with elastic ankle exoskeletons. IEEE Transactions on Neural Systems \& Rehabilitation Engineering, 2015, 63(5): 914-923. 